\title{
Cestrum yellow leaf curling virus (CmYLCV) promoter: a new strong constitutive promoter for heterologous gene expression in a wide variety of crops
}

\author{
Livia Stavolone ${ }^{1,5, *}$, Maria Kononova ${ }^{2}$, Sandra Pauli $^{1}$, Antonio Ragozzino ${ }^{3}$, Peter de Haan ${ }^{4,6}$, \\ Steve Milligan $^{2,7}$, Kay Lawton ${ }^{2}$ and Thomas Hohn ${ }^{1,8}$ \\ ${ }^{1}$ Friedrich Miescher Institute, Maulbeerstrasse 66, 4058 Basel, Switzerland; ${ }^{2}$ Syngenta Biotechnology, Inc., \\ 3054 Cornwallis Road, Research Triangle Park, NC 27709, USA; ${ }^{3}$ Istituto Patologia Vegetale, Università di \\ Napoli, 80055 Portici (NA), Italy; ${ }^{4}$ Syngenta, Enkhuizen, Netherlands; present addresses: ${ }^{5}$ Istituto di Virologia \\ Vegetale-CNR, via Amendola 165/A, Bari, Italy (*author for correspondence; e-mail l.stavolone@area.ba.cnrit); \\ ${ }^{6}$ Phytovation B.V., Wassenaarseweg 72, 2333 AL Leiden, Netherlands; ${ }^{7}$ Rosetta Inpharmatics, LCC 12040, 115th \\ Avenue NE, Kirkland, WA 98034, USA; ${ }^{8}$ Present address: University of Basel, Botanical Institute, Plant Health \\ Unit, Schoenbeinstrasse 6, CH-4056, Basel
}

Received 5 September 2003; accepted in revised form 5 November 2003

Key words: cestrum yellow leaf curling virus, constitutive expression, heritable expression, promoter, meristems, monocot and dicot expression, stable expression

\begin{abstract}
Appropriately regulated gene expression requires a suitable promoter. A number of promoters have been isolated and shown to be functional in plants, but only a few of them activate transcription of transgenes at high levels constitutively. We report here the cloning and characterization of a novel, constitutively expressed promoter isolated from Cestrum yellow leaf curling virus (CmYLCV), a double-stranded DNA plant pararetrovirus belonging to the Caulimoviridae family. The CmYLCV promoter is highly active in callus, meristems and vegetative and reproductive tissues in Arabidopsis thaliana, Nicotiana tabacum, Lycopersicon esculentum, Zea mays and Oryza sativa. Furthermore, the level of expression is comparable to, or higher than, that from the CaMV 35S, the 'superpromoter' or the maize ubiquitin 1 promoters, three frequently used promoters in agricultural biotechnology. The heritable, strong and constitutive activity in both monocotyledonous and dicotyledonous plants, combined with the extremely narrow $\mathrm{CmYLCV}$ host range, makes the CmYLCV promoter an attractive tool for regulating transgene expression in a wide variety of plant species.
\end{abstract}

\section{Introduction}

A major goal of agricultural biotechnology is to produce crops with enhanced agronomic traits such as disease and insect resistance, herbicide tolerance and abiotic stress tolerance, and with novel traits such as antibody production and enhanced nutritional value. For the genes directing these traits to be functional, they must be appropriately expressed.

A number of constitutive promoters have been isolated and tested in plants. However, only a few are being used in agricultural biotechnology (US Food and
Drug Administration Center for Food Safety and $\mathrm{Nu}-$ trition, http://www.cfsan.fda.gov/ lrd/biocon.html), and those currently available have limitations that narrow their utility. Furthermore, as genetic engineering of plants increases in complexity, there is an increased need for versatile promoters to direct expression of desirable transgenes in plants.

The most widely used, and perhaps best characterized, constitutive promoter is the $35 \mathrm{~S}$ promoter from cauliflower mosaic virus (CaMV) (Fang et al., 1989; Benfey et al., 1990), the type member of the Caulimoviridae family. The CaMV $35 \mathrm{~S}$ promoter drives 
high, mostly constitutive, levels of gene expression. However, when more than one copy of the promoter is present in the genome (Mette et al., 1999) or when plants are infected with CaMV (Al Kaff et al., 2000), the activity of this promoter may be decreased. This sensitivity to homology-dependent gene silencing (Matzke and Matzke, 1995) limits its utility. Another frequently used, highly expressed constitutive promoter is the synthetic transcription-regulating element known as the 'super-promoter', which consists of a trimer of the Agrobacterium ocs activator linked to the Agrobacterium mas activator plus promoter $(\mathrm{Ni}$ et al., 1995). This promoter drives expression of the $\beta$ glucuronidase reporter gene (gusA) in dicotyledonous plants at levels higher than the enhanced CaMV 35S promoter in almost all tissue types tested (Ni et al., 1995). However, the levels of expression of the 'superpromoter' in transgenic maize plants are much lower than in tobacco (Kononova et al., abstract in 20th Crown Gall Conference, Houston, TX, 1999), suggesting that this promoter is not very useful for the regulation of transgenes in monocotyledonous crops. A third highly active constitutive promoter is the Ubi1 promoter from the Zea mays polyubiquitin 1 gene (Christensen et al., 1995), which directs reporter gene expression in maize protoplasts to levels over tenfold higher than those from the CaMV 35S promoter. However, when expressed transiently in tobacco protoplasts the activity level of the Ubi-1 promoter drops drastically, indicating its monocot-specific expression (Christensen et al., 1995).

CmYLCV is a newly described member of the Caulimoviridae family (GenBank accession number AF364175) first isolated from Cestrum parqui, a plant belonging to the Solanaceae family (Ragozzino, 1974). Like other members of the Caulimoviridae, CmYLCV possesses a dsDNA genome replicated via a pregenomic/polycistronic RNA (Rothnie et al., 1994) that is transcribed under the control of the genomic full-length transcript promoter. Here, we report the heritable and stable ability of the CmYLCV promoter to drive strong, constitutive expression of heterologous genes in both dicot and monocot plant species. The CmYLCV promoter sequence is available from Syngenta Biotechnology, Inc. (www.syngentabiotechnology.com) for limited research purposes.

\section{Materials and methods}

\section{Plasmid construction}

Two basic plasmids were produced for transient assays, containing either the gusA or cat reporter genes terminated by the CaMV poly(A) signal. Both genes were placed under the control of two promoter fragments of different length from CmYLCV (CmpC and CmpS) or from CaMV 35S (PCa and PCaS1). The cassettes were cloned in a pUC18 vector (Stratagene) (details on all constructs described in the MS are available upon request).

For transformation of $A$. thaliana cv. Columbia, CmpCgusA and PCaS1 gusA cassettes were introduced into a modified pCAMBIA binary vector (Roberts et al., 2003) containing the bar gene selectable marker driven by Cochliobolus heterostrophus promoter 1 (P1) and terminated by the $35 \mathrm{~S}$ terminator (Figure 3A).

Three binary plasmids containing the CmpCsyngfpi, CmpSsyngfpi or CmpSgig fusion were constructed for Agrobacterium-mediated transformation of $Z$. mays and $O$. sativa plants. The syngfpi reporter gene is a monocot codon-optimized green fluorescence protein gene with the ST-LS1 intron from Solanum tuberosum (Vancanneyt et al., 1990). The synGFPI is assumed to localize to the cytoplasm as it does not contain a nuclear localization signal (NLS) or an ER retention sequence. gig is the $\beta$-glucuronidase (gusA) gene with ST-LS1 intron. CmpC and $\mathrm{CmpS}$ fragments were cloned into syngfpi-nopaline synthase (nos) terminator (Bevan et al., 1983) or gignos-containing plasmids to create the promoter-reporter gene fusions. These promoter-reporter cassettes were cloned into a binary vector containing a selectable marker cassette consisting of the Z. mays ubiquitin1 (Christensen et al., 1995) promoter linked to the pmi coding sequence from Escherichia coli (Negrotto et al., 2000) followed by the $3^{\prime}$ nos poly(A) addition sequence. For tomato transformation, the CmpCsyngfpi cassette was inserted into a binary vector containing a selectable marker cassette comprising the $\mathrm{CmpS}$ promoter operatively linked to pmi. The reporter-promoter cassette was inserted closest to the right border in all of the binary vectors produced.

A binary plasmid containing a ZmUbi1-syngfpi$35 \operatorname{Spoly}(\mathrm{A})$ cassette was used for comparison in maize. A binary vector containing the 'superpromoter'-syngfpi-nos and 'super-promoter'-pmi-nos cassettes was used for comparison in tomato. Bin- 
ary plasmids were introduced into Agrobacterium via electroporation.

\section{Transient expression}

Protoplast preparation and polyethylene glycol (PEG)mediated transfection of O. sativa, Nicotiana plumbaginifolia and C. parqui were performed as described previously (Goodall et al., 1990; Chen et al., 1996). Orychophragmus violaceus protoplasts were prepared and transfected by electroporation as described by Fütterer et al. (1989). For all types of protoplasts, $1-5 \mu \mathrm{g}$ of test plasmids were routinely co-transfected together with a distinct plasmid used as an internal standard control for transfection efficiency. GUS and CAT expression levels were determined upon protoplast cultivation and harvesting (Pooggin et al., 2000).

\section{Transgenic plant production}

Transgenic Z. mays plants were generated by Agrobacterium-mediated transformation of immature maize embryos essentially as described by Negrotto et al. (2000).

Transgenic $O$. sativa plants were generated by Agrobacterium-mediated transformation of embryogenic cultures generated from mature embryos. Mannose selection was carried out as described in Reed et al. (2001). Transformation of L. esculentum cotyledon hypocotyls was performed essentially as described in Fillati et al. (1987) with modifications for mannose selection by M. Sigareva (personal communication).

The transgenic virus-resistant tobacco plants were produced by Agrobacterium-mediated transformation with the Bin 19 binary vector system. Transgenic A. thaliana plants were produced by the flower-dip method (Clough and Bent, 1998) and resistant lines selected by resistance to the herbicide BASTA.

\section{Histochemistry}

For the histochemical detection of GUS activity, fresh plant tissue was incubated up to $48 \mathrm{~h}$ at $37^{\circ} \mathrm{C}$ in GUS reaction buffer containing $1 \mathrm{mM} \mathrm{X}$-gluc as substrate (Jefferson et al., 1987). After staining, samples were cleared by several washes with $70 \%$ ethanol.

\section{TaqMan copy number assay}

A quantitative multiplexed PCR reaction was used to determine the copy number of syngfpi and pmi coding sequences in maize and tomato transgenic events. Genomic DNA for the assays was isolated from young leaves of maize or tomato transgenic and non-transgenic plants. For a maize endogenous control, primers and probes were designed specific to the Z. mays alcohol dehydrogenase 1 (adhl) gene (GenBank accession number AF044295). For the tomato endogenous control, primers and probes were designed specific to the L. esculentum superoxide dismutase (sod) gene (GenBank accession number AF034411). For the transgenes, primers and probes were designed specific to the syngfpi (Syngenta) and pmi (GenBank accession number M15380) reporter genes. Transgene copy number was calculated by utilizing the exported cycle threshold $\left(C_{\mathrm{t}}\right)$ values basically as described previously (Ingham et al., 2001).

\section{SynGFPI and PMI ELISA}

SynGFPI protein concentration in non-transformed and transgenic plants was measured by indirect sandwich enzyme-linked immunosorbent assay (ELISA) with rabbit anti-GFP antibody (Torrey Pines Biolabs, TP401) for plate coating, goat anti-GFP (Rockland, 600-101-215) as secondary antibody and donkey anti-goat conjugated to alkaline phosphatase (Jackson ImmunoResearch, West Grove, PA) for detection. Absorbance was measured at $405 \mathrm{~nm}$ with $492 \mathrm{~nm}$ as reference on a microplate reader (Tecan Rainbow, Research Triangle Park, NC). The values were normalized to fit a standard four-parameter curve to plot GFP concentration versus absorbance. The same protocol was carried out for quantitative detection of PMI with goat anti-PMI antibody (Immunology, Syngenta)-coated plates, rabbit anti-PMI secondary antibody (Immunology, Syngenta) and a donkey antirabbit antibody conjugated to alkaline phosphatase (Jackson ImmunoResearch) for detection.

\section{Fluorescence microscopy}

For detection of syngfpi gene expression in the plant material, histological fluorescence microscopy analysis was performed. Tissue samples were collected from various tissue types of transgenic and nontransgenic maize and tomato plants. For detection of syngfpi gene expression, tissue samples were examined under a fluorescence stereomicroscope (Leica MZ FLIII) with the following filter set: excitation filter $470 / 40 \mathrm{~nm}$, barrier filter $525 / 50 \mathrm{~nm}$. No autofluorescence was detected with these filters. 


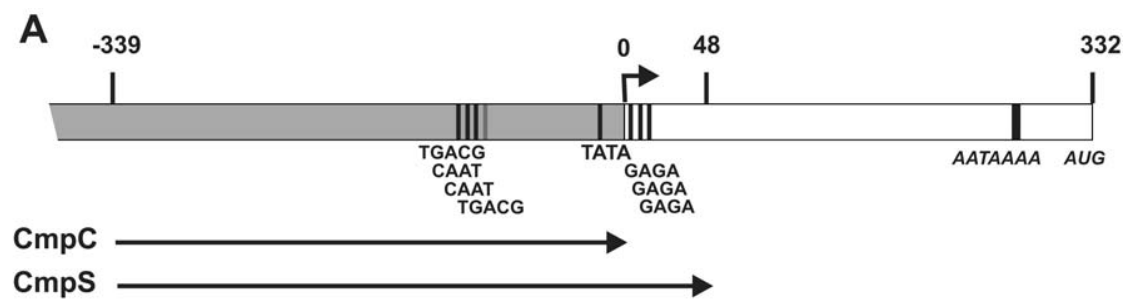

B

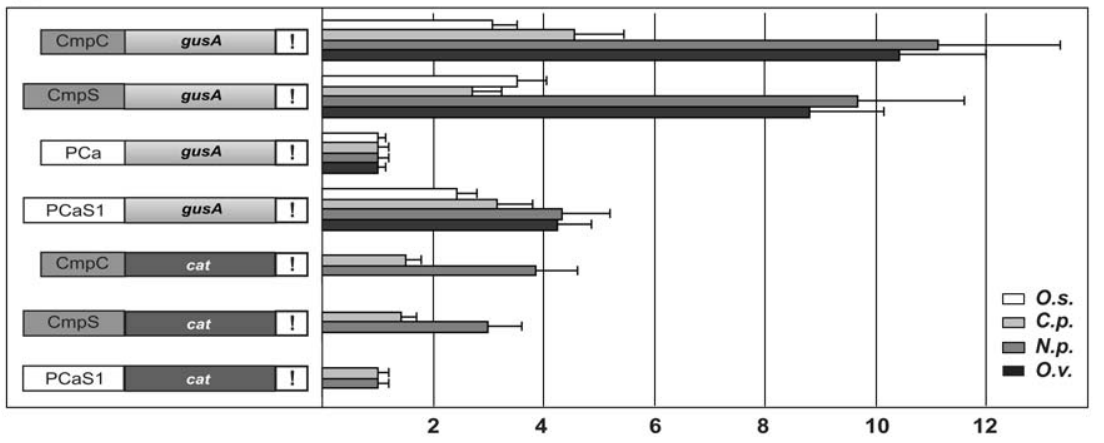

Figure 1. Structure and transient expression analysis of the CmYLCV promoter/leader region. A. Structure of the CmYLCV promoter/leader region. Promoter motifs (TGACG, CAAT, TATA and GAGA boxes) are indicated by vertical lines. The transcription start site is shown by a bent arrow. The positions of the polyadenylation sequence (AATAAA) and the start codon of the first large ORF (ORF1, AUG) are indicated. The two different length fragments selected from the $\mathrm{CmYLCV}$ promoter $(\mathrm{CmpC}$ and $\mathrm{CmpS})$ are illustrated by arrows. B. Transient expression of reporter genes under the control of CmYLCV or CaMV promoter fragments. Left panel: schematic representation of the expression cassettes. Two different length fragments from the CmYLCV (CmpC and CmpS) or CaMV 35S (PCa and PCaS1) promoter drive the gusA or cat reporter genes, both terminated by the CaMV poly(A) (!). Right panel: relative GUS and CAT activities in transient expression assays in protoplasts of different plant species: O.s., Oryza sativa; C.p., Cestrum parqui; N.p., Nicotiana plumbaginifolia; O.v., Orychophragmus violaceus. All GUS activities are expressed relative to values obtained with promoter PCa (set at 1.0); CAT activities are similarly expressed relative to the PCaS1-cat construct.

\section{Results and discussion}

\section{Structure of the CmYLCV promoter sequence}

The 8253 bp double-stranded DNA genome of CmYLCV has been cloned and sequenced (Stavolone et al., 2003). Analysis of the CmYLCV genomic sequence revealed the presence of an AT-rich region within the $671 \mathrm{bp}$ intergenic region containing a putative TATA box sequence (TATAAATA) (6395-6402) $5^{\prime}$ of the translation start site of ORF1, which begins at 6756 of the genomic sequence (Figure 1). Based on this information, we predicted the location of a putative transcription start site $32 \mathrm{bp} 3^{\prime}$ of this TATA box at 6426 (counted from the first T of this box). A comparison with other pararetrovirus promoter sequences revealed very low sequence similarity, limited to highly conserved motifs (Gardner et al., 1981; Medberry et al., 1992; Verdaguer et al., 1996). They are all located $5^{\prime}$ of the TATA box and include two as1-like enhancer elements (TGACG, 6254-6258, 6333-6337) and two putative CAAT boxes (6319-6322 and 6330-6333).

The promoter region also contains three potential binding sites for transcription modulation factors located within the region +8 to +35 relative to the predicted transcription start site. They are known as GAGA motifs and shown to be functional in the equivalent region of other pararetroviral promoters $(\mathrm{He}$ et al., 2002; Pauli et al., submitted). A 330 bp leader sequence separates the site of transcription initiation from the translation start site. As in other reversetranscribing viruses (see Rothnie et al., 1994), a conditional poly(A) signal (AATAAAA, 6687-6693) is located within the leader region.

Viral sequences located between the transcription and translation start sites have been found to stimulate expression of a downstream reporter gene (Day Dowson et al., 1993; Chen et al., 1996). Therefore two CmYLCV promoter fragments were selected for analysis, a shorter 346 bp fragment termed $\mathrm{CmpC}$ (6088-6433) and a longer $400 \mathrm{bp}$ fragment termed 
A

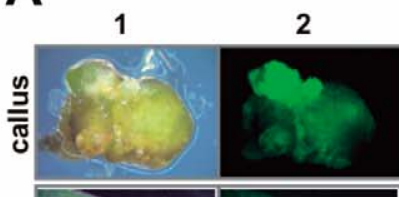

3

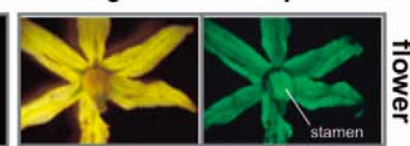

$\stackrel{\square}{\Xi}$
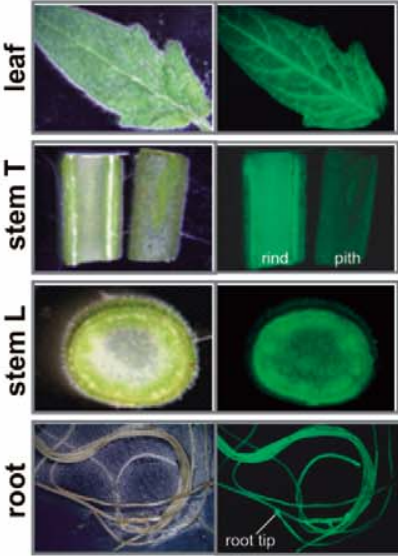

syngfpi-transformed
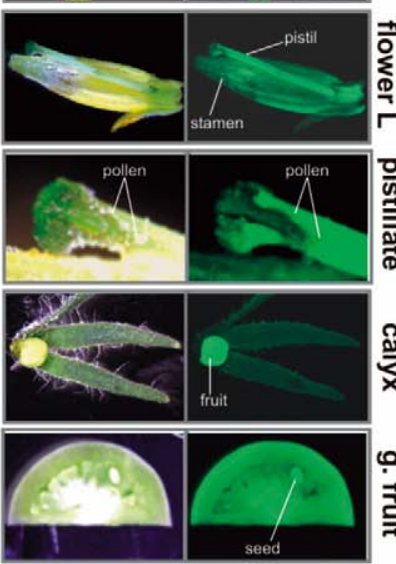

ed

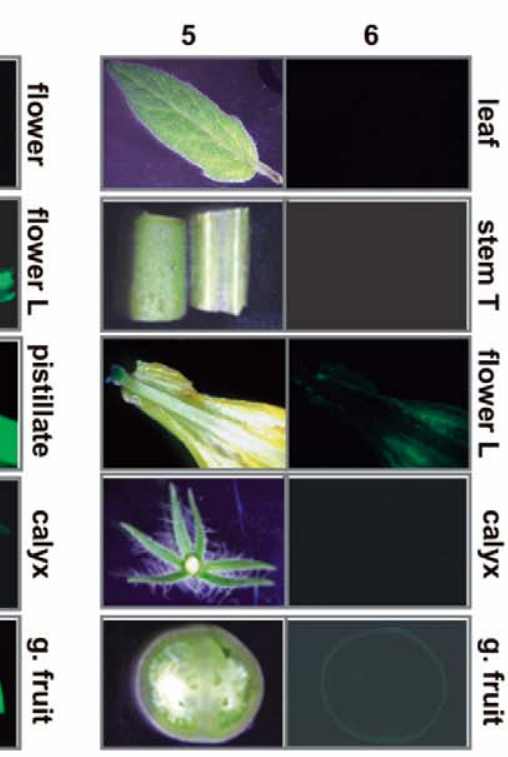

Non-transformed

B

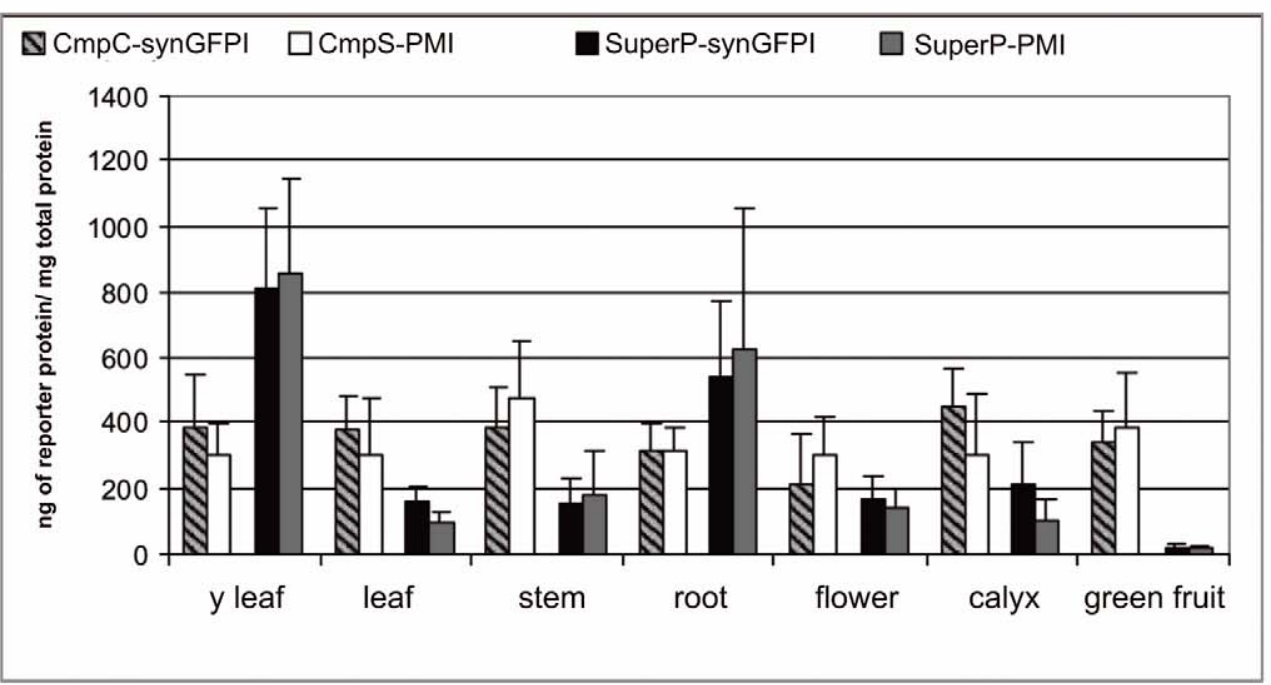

Figure 2. The CmYLCV promoter is highly active in transgenic tomato. A. Representative images from single-copy tomato lines transformed with a CmpCsynGFPI fusion and non-transgenic tomato, under white light (columns 1,3 and 5) and UV illumination (columns 2, 4 and 6). L, longitudinal section; T, transverse section; g. fruit, green immature tomato fruit. B. Comparative expression of the syngfpi and pmi reporter genes under the control of the $\mathrm{CmpC}$, the $\mathrm{CmpS}$ and the 'super-promoter' (SuperP) promoters in the $\mathrm{T}_{0}$ generation. On average, fifteen independent single-copy transgenic lines were analyzed for each construct. Y leaf represents young leaves harvested from tissue culture vessels prior to transplanting to soil. Neither synGFP nor PMI was detectable above background in non-transgenic plants (data not shown). 
CmpS (6088-6487) (Figure 1). The CmpC promoter fragment contains the entire essential promoter motifs described above and ends at +8 (relative to the transcription start site). The $\mathrm{CmpS}$ promoter fragment extends $62 \mathrm{nt}$ downstream of the transcriptional start site into the leader sequence.

\section{The CmYLCV promoter is highly active in transient expression assays}

To assess the activity of the CmYLCV promoter, fragments with $(\mathrm{CmpS})$ or without $(\mathrm{CmpC})$ the leader sequence were cloned $5^{\prime}$ of either the gusA or the chloramphenicol acetyltransferase (cat) reporter genes. As a first approach, the level of reporter gene expression under the control of each of these two CmYLCV promoter fragments was determined in transiently transfected protoplasts from different plant species, and compared to levels obtained with two versions of the CaMV 35S promoter: $\mathrm{PCa}(-227$ to +8 relative to the transcription start site) and $\mathrm{PCaS} 1$ $(-227$ to +56$)$, i.e. similar in size to $\mathrm{CmpC}$ and CmpS, respectively. Results obtained from ten independent experiments are illustrated in Figure 1B. These results show that GUS activity under the control of the CmYLCV promoter was up to more than ten times higher than that obtained under control of the PCa promoter in Nicotiana plumbaginifolia and Orychophragmus violaceus protoplasts, and 3-5 times stronger in Oryza sativa and in C. parqui protoplasts. The cat gene was also expressed at a higher level when driven by the CmYLCV promoter, although a smaller difference was observed than with the gusA reporter (Figure 1B). In both GUS and CAT assays, the activity of the $\mathrm{CmpC}$ promoter fragment was the same as, or more than, that of $\mathrm{CmpS}$, indicating that the first 62 bases of the CmYLCV leader had no stimulatory effect on promoter activity. This is in contrast to results obtained with CaMV, where the presence of the corresponding region of the 35S RNA leader sequence results in increased reporter gene expression (Figure 1B, Fütterer et al., 1990).

\section{CmYLCV promoter drives strong transgene expression in dicots}

To be a useful tool for plant biotechnology, a promoter must function in agronomically significant crop species. The CmYLCV promoter was evaluated in transgenic tomato plants transformed with the $\mathrm{CmpC}$ promoter fragment fused to a synthetic, intron-containing, monocot codon-optimized green fluorescence protein (syngfpi). The results of detailed analysis of transgenic tomato tissues performed on single-copy events to determine the tissue-specific pattern of CmYLCV expression are shown in Figure 2A. Histological evaluation revealed that the CmYLCV promoter was functional in all vegetative organs analyzed, including leaves, root tips, stem branch primordium and cambium. The promoter was also highly active in all reproductive organs except pollen, where only very low levels of activity were detected. In addition, strong expression of the CmYLCV promoter in vegetative tomato callus was observed (Figure 2A).

To quantify the level of promoter activity, the $\mathrm{CmpC}$ and $\mathrm{CmpS}$ fragments were evaluated relative to the strong, constitutive 'super-promoter' in transgenic tomato plants. Binary vectors used for L. esculentum transformation differed only in the promoter used to drive the expression of either the syngfpi or the phosphomannose isomerase ( $p m i)$ gene. A sensitive and highly reproducible ELISA was developed for measuring the levels of PMI and synGFPI, thus both proteins could be used as reporters of promoter activity. Figure $2 \mathrm{~B}$ shows the results of analysis of the CmYLCV and the 'super-promoter' promoter activity in several independent, single copy transgenic tomato lines. Both CmYLCV promoter fragments drive the expression of both syngfpi and pmi at similar levels in all tissue types tested, suggesting that the nature of the coding sequence does not influence the activity of this promoter. Further, syngfpi and pmi expression driven by either of the CmYLCV promoter fragments was 2-3 times higher than the level obtained with the 'super-promoter' in leaf, stem, flower and calyx. In green fruit, the average activity of the CmYLCV promoter was 20 -fold higher than that observed with the 'super-promoter', while the CmYLCV promoter was weaker than the 'super-promoter' in roots. This might be explained by the nature of the synthetic 'super-promoter', which is comprised of promoter and activator elements of genes from the soil-borne pathogen, Agrobacterium tumefaciens (Ni et al., 1995). Interestingly, the 'super-promoter' was stronger than the CmYLCV promoter in young leaves of primary transformants on root-inducing media, but significantly lower in the leaves of mature plants grown in soil in the greenhouse (Figure 2B, y-leaf vs. leaf). In contrast, the CmYLCV promoter showed similar levels of expression in leaves of plants from different developmental stages, suggesting that CmYLCV promoter activity may be independent of plant growth conditions and/or developmental stage. Activity of 

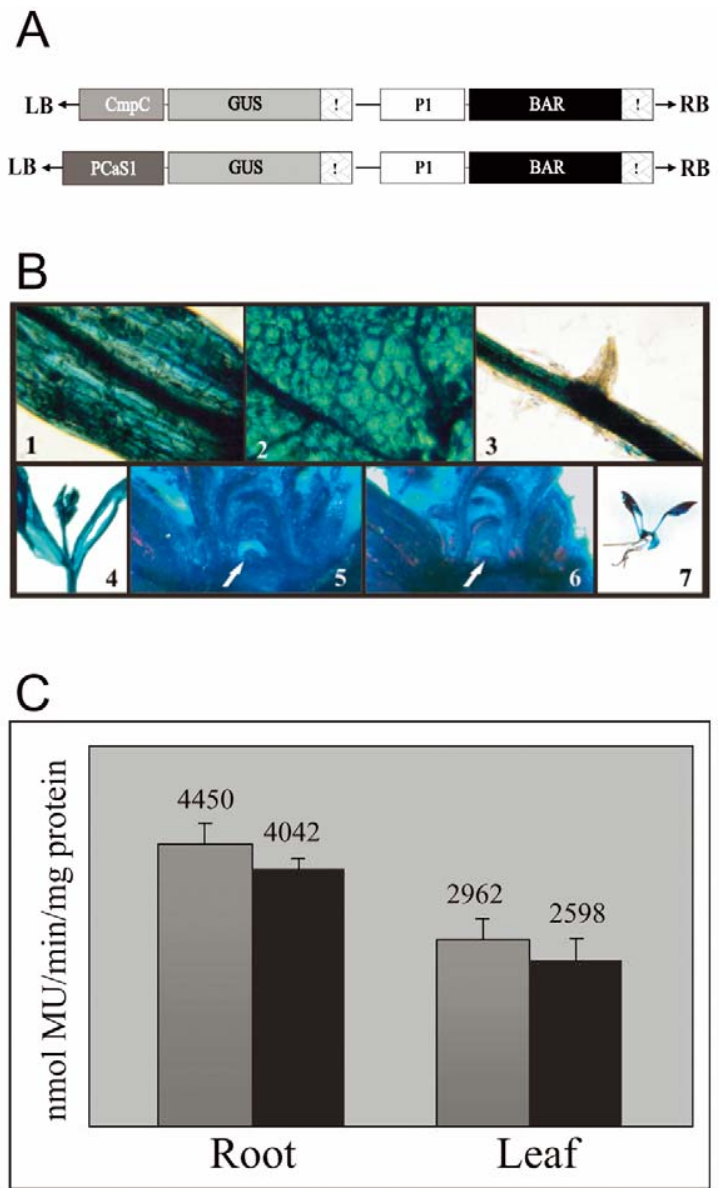

Figure 3. Analysis of GUS expression in transgenic A. thaliana. A. Transgene constructs used for plant transformation. The gusA reporter gene is under the control of either the CmYLCV CmpC or the CaMV PCaS1 promoter. B. Histochemical analysis of GUS expression in transgenic $A$. thaliana: 1, stem; 2, leaf; 3, root; 4, flower; 7, whole plant transformed with the PCmpCgusA construct. Sections of A. thaliana inflorescences showing apical meristems (white arrows) transformed with PCmpCgusA (5) and PCaS1gusA (6). C. Fluorometric analysis of GUS enzymatic activities in roots and leaves. Data shown are the average activities of 8 independent $\mathrm{T}_{2}$ generation lines under the control of the CmpC promoter (gray columns) and 6 under PCaS1 control (black columns). 8 to 10 individual plants per line were analyzed.

the CmYLCV promoter was also evaluated in the $\mathrm{T}_{1}$ generation of tomato to determine stability of expression in the next generation. Both promoter fragments retained high levels of expression in $\mathrm{T}_{1}$ plants, showing that the high constitutive expression from the CmYLCV promoter is heritable (data not shown).

Because of the importance of $A$. thaliana in present plant genetics, we also analyzed the expression levels of the CmYLCV promoter in comparison with PCaS1, the stronger of the two CaMV 35S promoter fragments tested (Figure 1, Pauli et al., submitted), in this plant (Figure 3A). Single-copy insert lines of the $\mathrm{T}_{2}$ generation were selected to test GUS activity. Histochemical GUS staining analysis performed on whole plants transformed with $\mathrm{CmpCgusA}$ indicated constitutive and stable expression in all organs investigated. Unlike CaMV35S, the CmYLCV promoter drives gusA expression also in flower meristems (Figure 3B). Quantitative GUS fluorometric assays were performed on leaves and roots. Similar levels of GUS expression were detected with $\mathrm{CmpC}$ and PCaS1 promoters (Figure 3C). The GUS expression level was higher in roots than in leaves regardless of which promoter was used (Figure 3C).

\section{The CmYLCV promoter is active at high levels constitutively in monocots}

To determine if CmYLCV promoter activity extends to agronomically important monocotyledonous crop species, transgenic $Z$. mays and $O$. sativa, two of the 
most important agronomic crops, were generated. Two different reporter genes, syngfpi in maize and gusA in rice, were used to monitor promoter expression patterns in different tissue types. Detailed histological analysis of transgenic maize and rice tissues was performed on single-copy events by fluorescence or light microscopy respectively. The CmYLCV promoter was highly active in both maize (Figure 4A) and rice (Figure 4B). Non-transgenic plants did not show any autofluorescence under UV illumination (Figure 4A, column 6). High levels of reporter gene expression were observed in all vegetative and reproductive organs tested, except pollen. These observations are consistent with the expression patterns observed in transgenic L. esculentum plants (Figure 2A). Histological analysis of transgenic maize and rice seeds revealed strong expression of the CmYLCV promoter in both endosperm and embryo. Importantly, strong reporter gene expression was observed in embryogenic callus and, like in tomato and A. thaliana, in meristematic cells such as root tips, cambium and flower meristems, suggesting that the promoter will be useful for early selection of transgenic plants (Figure 4).

In maize, the $\mathrm{CmpC}$ and $\mathrm{CmpS}$ promoter fragments were compared to the robustly expressed constitutive maize Ubil promoter. Depending on the tissue type tested, the CmYLCV promoter drives reporter gene expression to levels as much as twenty times higher than those observed with the Ubi1 promoter (Figure 4C). The lowest level of CmYLCV promoter activity was detected in pollen. This may potentially confer an ecological advantage on the use of this promoter with regard to protection of pollenfeeding butterflies when expressing insect resistance genes. Furthermore, in all tissue types available for the test, expression levels driven by the $\mathrm{CmpC}$ and the $\mathrm{CmpS}$ promoter fragment were similar. This is consistent with results obtained both in transient assays and in whole tomato plants, and supports the conclusion that the presence of a short (62 nt) leader sequence does not influence the expression level of the $\mathrm{CmpC}$ promoter fragment. Taken together, these results demonstrate that the CmYLCV promoter is a functional, constitutive and highly expressed promoter useful for regulating the expression of heterologous genes in monocotyledonous crop plants.
CmYLCV promoter activity is stable and heritable, with low susceptibility to gene silencing

A critical factor determining promoter utility in transgenic crops is its ability to retain expression level and functionality over multiple generations and under field conditions. To test whether CmYLCV promoter activity is heritable, CmYLCV promoter activity was evaluated in the $T_{1}$ generation of single-copy lines. As shown in Figure 5A, the level of reporter gene expression measured in hemizygous $T_{1}$ plants was similar to levels measured in $\mathrm{T}_{0}$ plants. Further, when reporter gene expression levels in hemizygous and homozygous $\mathrm{T}_{1}$ maize plants were compared, promoter activity was higher in all of the homozygous plants tested (Figure 5A). Activity of the CmYLCV promoter was also evaluated in the $T_{1}$ generation of tomato. Both promoter fragments retain high expression levels in $\mathrm{T}_{1}$ tomato plants (data not shown). These results show that the high constitutive expression from the CmYLCV promoter is heritable.

Experience has shown that viral promoters are prone to transcriptional gene silencing (TGS) (Matzke and Matzke 1995; Al Kaff et al., 2000), which depends not only on the strength of a promoter but can also be initiated by the presence of multiple copies of homologous transgenes and/or promoters, leading to DNA methylation (Mette et al., 1999), heterochromatization (Soppe et al., 2002) and cessation of transcription from all cognate promoters. To analyze this further, the influence of gene copy number on CmYLCV promoter activity was evaluated in the $\mathrm{T}_{0}$ generation of transgenic maize lines. On average, CmYLCV promoter activity was higher in lines containing two or more copies of the reporter gene than in single copy maize lines (Figure 5B). Thus, our results suggest that it is unlikely that transgene silencing will be triggered by the presence of multiple copies of the CmYLCV promoter in the transgenic plant.

A single copy of a transgene driven by a viral promoter may also be susceptible to virus-induced gene silencing (VIGS) upon infection of the transformed plant with the virus (Ruiz et al., 1998; Al Kaff et al., 2000). This was observed for CaMV, which is infectious to members of the Solanaceae, Resedaceae and most Cruciferae plant families. Of a wide range of test plants and commercially important crop species evaluated by mechanical inoculation, CmYLCV infected only C. parqui, C. elegans and Nicotiana clevelandii (Stavolone et al., 2003). This suggests that a crop plant transformed with the CmYLCV promoter has a high 


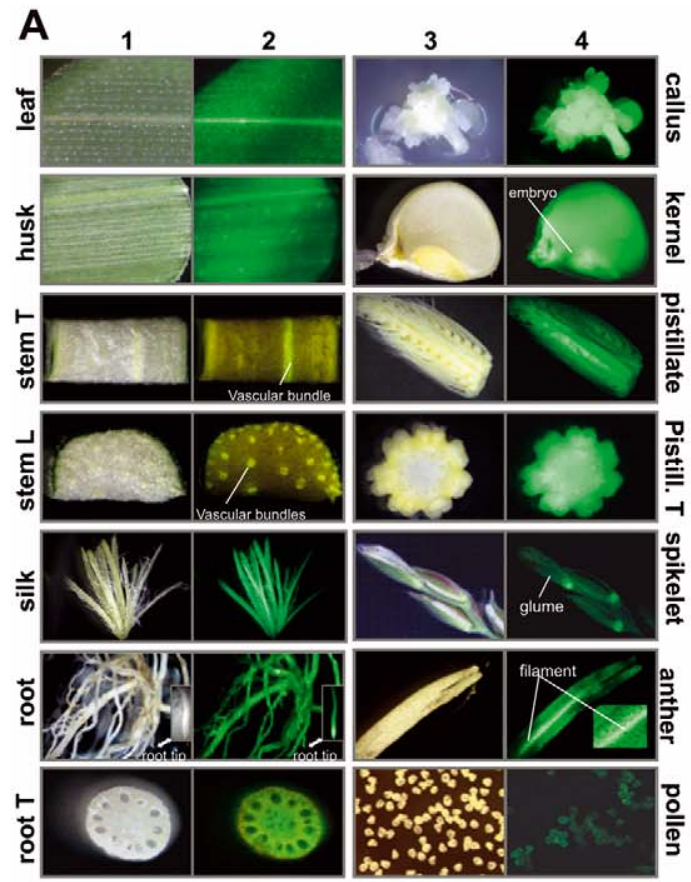

syngfpi- transformed

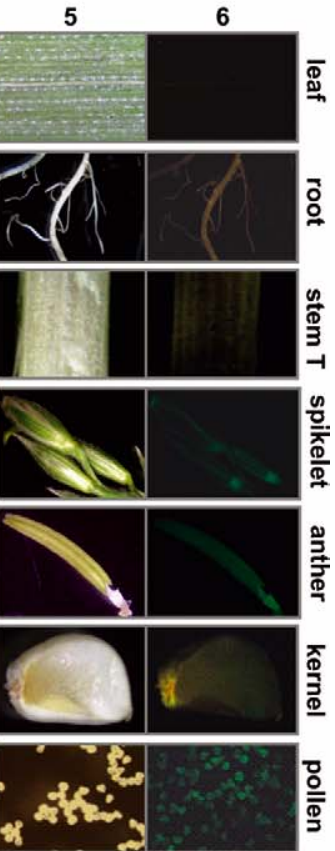

Non- transformed

B
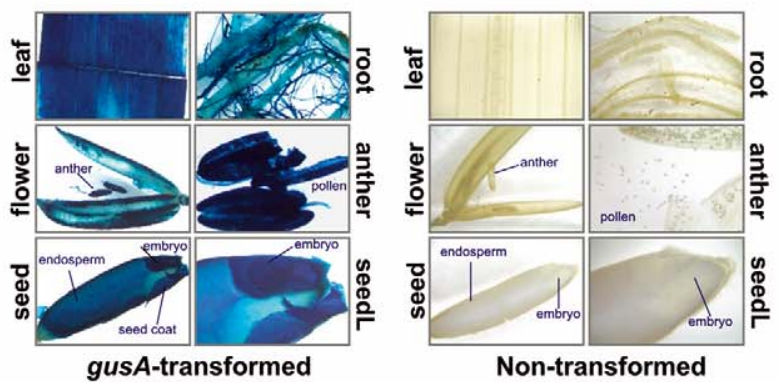

Non-transformed

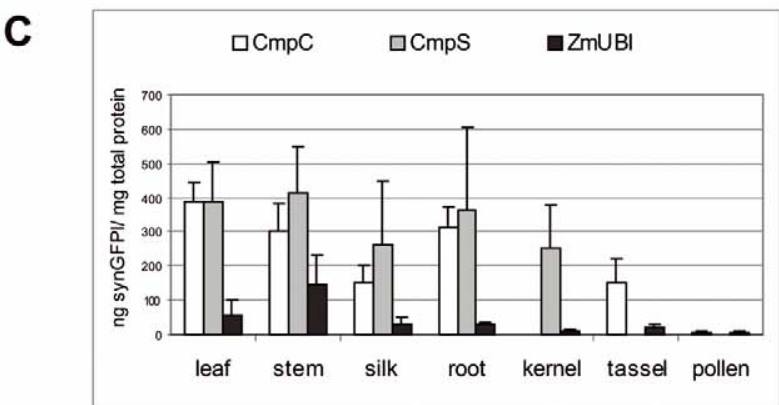

Figure 4. The CmYLCV promoter is highly active in transgenic monocots. A. Representative images from single-copy $\mathrm{T}_{0}$ maize (Zea mays) lines transformed with either CmpCsyngfpi or CmpSsyngfpi fusions and from non-transgenic maize under white light (columns 1, 3 and 5) or UV illumination (columns 2, 4 and 6). L, longitudinal section; T, transverse section; pistil., pistillate. B. Representative images from single-copy $\mathrm{T}_{0}$ rice (O. sativa) lines transformed with CmpSgusA fusion and from non-transformed control plants. Images correspond to $48 \mathrm{~h}$ $\mathrm{X}$-Gluc-stained plant material. GUS staining in all transgenic tissues except pollen was apparent at $1.5 \mathrm{~h}$ of incubation in X-Gluc (not shown) but it was not observed in non-transgenic plants stained for $48 \mathrm{~h}$ (right panel). C. Comparative synGFPI expression under the control of CmpC, $\mathrm{CmpS}$ and maize Ubi1 promoters in various $\mathrm{T}_{0}$ generation maize tissues. On average, twenty independent transgenic single-copy $\mathrm{T}_{0}$ lines were analyzed for each construct by ELISA test. Kernels of CmpC-transformed lines and tassels and pollen of CmpS lines were not available for the test. No expression above background levels was detected in non-transgenic control plants (not shown). 

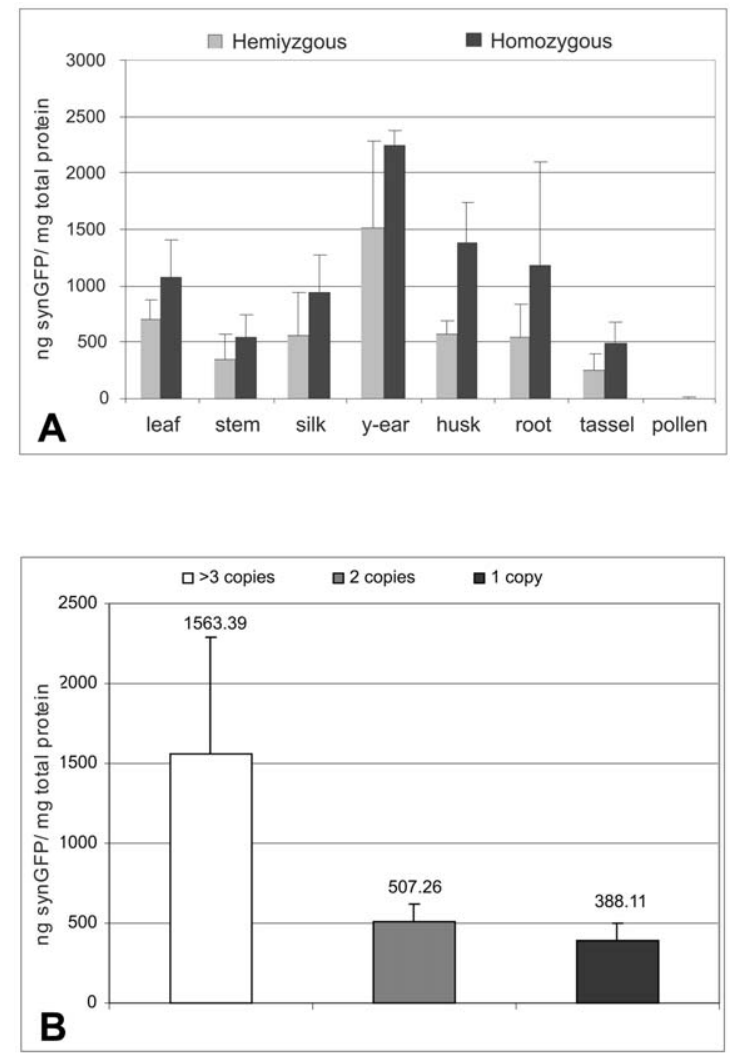

Figure 5. Influence of multiple transgene copy on CmYLCV promoter activity. A. Comparative expression of the CmpCsyngfpi transgene in different tissues of homozygous and hemizygous $\mathrm{T}_{1}$ maize plants. Eleven independent homozygous and hemizygous lines (five plants per line) were used for the ELISA. y-ear, immature ear (pistillate flower). B. Expression levels of the CmpSsyngfpi transgene in leaves of single- and multiple-copy $\mathrm{T}_{0}$ maize lines. Each bar represents the average expression of six two-copy, six multiple-copy ( 3 or more copies of the transgene) and fifteen single-copy independent transgenic lines.

chance of escaping silencing either upon CmYLCV infection or, due to the low sequence similarity with the $35 \mathrm{~S}$ promoter, upon CaMV infection.

\section{Conclusions}

Our results show that the CmYLCV promoter is a novel tool with broad utility for regulating the constitutive expression of transgenes at high levels in both monocot and dicot crops. The constitutive activity of the CmYLCV promoter is equal to or higher than the level obtained with any of the most commonly used constitutive promoters tested here. It is unlikely that the different activity of the promoters tested is the result of a transgene positional effect. In fact, we analyzed numerous independent single-copy transgene lines and measured comparable levels of activity with each particular promoter.

Interestingly, the CmYLCV promoter has high activity in meristematic cells and callus. This feature indicates that, in addition to being useful to direct high levels of gene expression in most cell and tissue types of a wide selection of crop plants, the CmYLCV promoter may also be useful for driving expression of a selectable marker gene for transgenic plant production. Importantly, our results show that the high expression levels are heritable, do not appear to be negatively influenced by multiple copies of the promoter and, because the virus has a narrow host range (Stavolone et al., 2003) and the promoter has low sequence similarity to other pararetroviruses, including, most notably, the CaMV $35 \mathrm{~S}$ promoter, gene silencing is predicted to be unlikely. Thus, the CmYLCV promoter is a new and truly useful tool for agricultural research and biotechnology applications.

\section{Acknowledgments}

We would like to thank Marina Sigareva, Jeff Bottoms, Vladimir Samoylov and Erik Dunder for help with production of transgenic tomato, maize and rice plants; Christina White, Dawn McNamara and Brian Potter for maintenance of transgenic plants; Michelle Yarnall for optimization of PMI and synGFI ELISA protocols; the Syngenta Plant Analysis group for help with TaqMan copy number assays; and Matthias Müller for protoplast preparation. We are grateful to Barbara Hohn and members of her lab for many valuable discussions and to Helen Rothnie for proofreading and suggestions. L.S. was partially supported by a grant from Novartis, now Syngenta and partially by an EMBO short-term fellowship.

\section{References}

Al Kaff, N.S., Kreike, M.M., Covey, S.N., Pitcher, R., Page, A.M. and Dale, P.J. 2000. Plants rendered herbicide-susceptible by cauliflower mosaic virus-elicited suppression of a $35 \mathrm{~S}$ promoterregulated transgene. Nature Biotech. 18: 995-999.

Benfey, P.N., Ren, L. and Chua, N.H. 1990. Combinatorial and synergistic properties of CaMV $35 \mathrm{~S}$ enhancer subdomains. EMBO J. 9: 1685-1696.

Bevan, M., Barnes, W.M. and Chilton, M.D. 1983. Structure and transcription of the nopaline synthase gene region of T-DNA. Nucl. Acids Res. 11: 369-385. 
Chen, G., Rothnie, H.M., He, X., Hohn, T. and Fütterer, J. 1996. Efficient transcription from the rice tungro bacilliform virus promoter requires elements downstream of the transcription start site. J. Virol. 70: 8411-8421.

Christensen, A.H., Sharrock, R.A. and Quail, P.H. 1992. Maize polyubiquitin genes: structure, thermal perturbation of expression and transcript splicing, and promoter activity following transfer to protoplasts by electroporation. Plant Mol. Biol. 18: 675-689.

Clough, S.J. and Bent, A.F. 1998. Floral dip: a simplified method for Agrobacterium-mediated transformation of Arabidopsis thaliana. Plant J. 16: 735-743.

Day Dowson, M.S., Ashurst, J.L., Mathias, S.F., Watts, J.W., Wilson, T.M.A. and Dixon, R.A. 1993. Plant viral leaders influence expression of a reporter gene in tobacco. Plant Mol. Biol. 23: 97-109.

de Haan, P., Gielen, J.J.L., Prins, M., Wijkamp, I.G., van Schepen, A.D., van Grinsven, M.Q.J.M. and Goldbach, R. 1992. Characterization of RNA-mediated resistance to tomato spotted wilt virus in transgenic tobacco plants. Bio/technology 10: 11331137.

Fang, R.X., Nagy, F., Sivasubramaniam, S. and Chua, N.H. 1989. Multiple cis regulatory elements for maximal expression of the cauliflower mosaic virus $35 \mathrm{~S}$ promoter in transgenic plants. Plant Cell 1: 141-150.

Fillati, J.J., Kiser, J., Rose, R. and Comai, L. 1987. Efficient transfer of a glyphosate tolerance gene into tomato using a binary Agrobacterium tumefaciens vector. Bio/technology 5: 726-730.

Fütterer, J., Gordon, K., Pfeiffer, P., Sanfaçon, H., Pisan, B., Bonneville, J.M. and Hohn, T. 1989. Differential inhibition of downstream gene expression by the cauliflower mosaic virus $35 \mathrm{~S}$ RNA leader. Virus Genes 3: 45-55.

Fütterer, J., Gordon, K., Sanfaçon, H., Bonneville, J.M. and Hohn, T. 1990. Positive and negative control of translation by the leader sequence of cauliflower mosaic virus pregenomic 35S RNA. EMBO J. 9: 1697-1707.

Gardner, R.C., Howarth, A.J., Hahn, P., Brown-Luedi, M., Shepherd, R.J. and Messing, J. 1981. The complete nucleotide sequence of an infectious clone of cauliflower mosaic virus M13mp7 shotgun sequencing. Nucl. Acids Res. 9: 2871-2887.

Goodall, G.J., Wiebauer, K. and Filipowicz, W. 1990. Analysis of pre-mRNA processing in transfected plant protoplasts. Meth. Enzymol. 181: 148-161.

He, X., Fuetterer, J. and Hohn, T. 2002. Contribution of downstream promoter elements to transcriptional regulation of the rice tungro bacilliform virus promoter. Nucl. Acids Res.30: 497-506.

Ingham, D.J., Beer, S., Money, S. and Hansen, G. 2001. Quantitative real-time PCR assay for determining transgene copy number in transformed plants. BioTechniques 31: 132-134, 136-140.

Jefferson, R.A. 1987. Assaying chimeric genes in plants: the GUS gene fusion system. Plant Mol. Biol. Rep. 5: 387-405.

Matzke, M.A. and Matzke, A.J. 1995. Homology-dependent gene silencing in transgenic plants: what does it really tell us? Trends Genet. 11: 1-3.

Medberry, S.L., Lockhart, B.E. and Olszewski, N.E. 1992. The Commelina yellow mottle virus promoter is a strong promoter in vascular and reproductive tissues. Plant Cell 4: 185-192.
Mette, M.F., van der Winden, W.J., Matzke, M.A. and Matzke, A.J. 1999. Production of aberrant promoter transcripts contributes to methylation and silencing of unlinked homologous promoters in trans. EMBO J. 18: 241-248.

Negrotto, D., Jolley, M., Beer, S. and Wenck, A.R. 2000. The use of phosphomannose-isomerase as a selectable marker to recover transgenic maize plants (Zea mays L.) via Agrobacterium transformation. Plant Cell Rep. 19: 798-803.

Ni, M., Cui, D., Einstein, J., Narasimhulu, S., Vergara, C. and Gelvin, S. 1995. Strength and tissue specificity of chimeric promoters derived from the octopine and mannopine synthase genes. Plant J. 7: 651-676.

Pooggin, M.M., Hohn, T. and Fütterer, J. 2000. Role of a short open reading frame in ribosome shunt on the cauliflower mosaic virus RNA leader. J. Biol. Chem. 275: 17288-17296.

Ragozzino, A. 1974. Una virosi del Cestrum parqui L'Erit (Fam. Solanaceae) in Campania. Ann. Fac. Sci. Agric. Univ. Napoli IV 8: 249.

Reed, J., Privalle, L., Powell, M.L., Dawson, J., Dunder, E., Suttie, J., Wenck, A., Launis, A., Kramer, C., Chang, Y.-F., Hansen, G. and Wright, M. 2001. Phosphomannose isomerase: an efficient selectable marker for plant transformation. In vitro Cell. Dev. Biol. Plant 37: 127-132.

Roberts, C.S., Rajagopal, S., Smith, L.A., Nguyen, T.A., Yang, W., Nugroho, S., Ravi, K.S., Vijayachandra, K., Harcourt, R.L., Dransfield, L., Desamero, N., Slamet, I., Hajdukiewic, P., Svab, Z., Maliga, P., Mayer, J.E., Keese, P., Kilian, K. and Jefferson, R.A. 2003. A comprehensive set of modular vectors for advanced manipulations and efficient transformation of plants by both Agrobacterium and direct DNA uptake methods. WEB-site: www.cambia.org. au/main/r_et_vman.htm

Rothnie, H.M., Chapdelaine, Y. and Hohn, T. 1994. Pararetroviruses and retroviruses: a comparative review of viral structure and gene expression strategies. Adv. Virus Res. 44: 1-67.

Ruiz, M.T., Voinnet, O. and Baulcombe, D.C. 1998. Initiation and maintenance of virus-induced gene silencing. Plant Cell 10: 937946.

Soppe, W.J., Jasencakova, Z., Houben, A., Kakutani, T., Meister, A., Huang, M.S., Jacobsen, S.E., Schubert, I. and Fransz, P.F. 2002. DNA methylation controls histone H3 lysine 9 methylation and heterochromatin assembly in Arabidopsis. EMBO J. 21: 6549-6559.

Stavolone, L., Ragozzino, A. and Hohn, T. 2003. Characterization of cestrum yellow leaf curling virus: a new member of the caulimoviridae family. J. Gen. Virol. 84: 3459-3464.

Vancanneyt, G., Schmidt, R., O'Connor-Sanchez, A., Willmitzer, L. and Rocha-Sosa, M. 1990. Construction of an intron-containing marker gene: splicing of the intron in transgenic plants and its use in monitoring early events in Agrobacterium-mediated plant transformation. Mol. Gen. Genet. 220: 245-250.

Verdaguer, B., de Kochko, A., Beachy, R.N. and Fauquet, C. 1996. Isolation and expression in transgenic tobacco and rice plants, of the cassava vein mosaic virus (CVMV) promoter. Plant Mol. Biol. 31: 1129-1139. 